\title{
Review, Hypothesis
}

The Meaning of the Microprocessor: Accounting for Evolution of Structural-Functional Novelty in the Canonical microRNA Biogenesis Pathway.

Jeffrey Robinson, PhD, MS.

Phone: 301-335-4851

Email: jeff.robinson.evol@gmail.com

Orcid: https://orcid.org/0000-0002-1456-2851

ResearchGate: https://www.researchgate.net/profile/Jeffrey Robinson4

Keywords: Microprocessor, miRNA, Drosha, Pasha, miRNA biogenesis pathway, Holozoa, Metazoa, RNA interference, bona fide miRNA, canonical eumetazoan miRNA.

\section{Abstract.}

It is standard to identify and compare predicted protein sequence of the Drosha and Pasha genes subsidiary to detection and identification of novel microRNAs in newly sequenced taxa or review of previous deep sequencing data. Drosha and Pasha proteins are the key, conserved members of the 'microprocessor' protein complex which facilitates nuclear nuclear-localized, pri- to pre-miRNA processing miRNAs of the canonical eumetazoan complement. Because of the necessity of the microprocessor for production of canonical eumetazoan miRNA, the detection of both (1) bona fide microRNAs and (2) presence of Drosha/Pasha orthologs (or homologs) is often presented as sufficient to represent a functional canonical eumetazoan microRNA biogenesis pathway. However, the functional role of the Drosha and Pasha homologs sometimes, though not always experimentally validated in non-model taxa. Differentiation of 'bona fide miRNAs', opposed to 'non-bona fide' small RNAs of similar size, are also necessary for miRNA identification projects. Recent rubrics are based on structural and sequence elements of the miRNAs themselves, however these inclusion criteria include paraphyletic groupings of miRNAs, for example eumetazoan miRNAs and Streptophyte (green plant) miRNAs which are not produced by the Drosha/Pasha microprocessor mechanism. Therefore, a dichotomy exists between the structural definitions for miRNAs and understanding of the evolutionarily conserved function of the microprocessor and its components. In this article, I review literature in the context of this topic and discuss philosophical significance for understanding the importance of the microprocessor in understanding the evolutionary and molecular origins of miRNA.

Drosha and Pasha orthology is not sufficient to assert shared evolutionary origin of canonical microRNA biogenesis for Eumetazoa, basal Metazoa, and Ichthyosporea, yet experimental validation of putative Drosha homolog in Dictyostelium may indicate even more deeply evolutionary origin of microprocessor.

Identification of Drosha and Pasha homologs is usually performed by sequence similarity using the most highly conserved sequence regions corresponding to the C-terminal RNAselll and dsRBD protein domains of Drosha and Pasha, and showing that Drosha and Pasha sequences are true homologs provides a basis for a canonical, microprocessor-based metazoan microRNA biogenesis pathway. The $\mathrm{N}$-terminal half of Drosha contains various putative protein-protein interaction domains, which while somewhat well conserved in Eumetazoa, appear divergent in basal metazoans from Porifera and Placozoa. Many sequence-based Drosha and Pasha comparisons 
for diverse metazoan taxa are found in the literature (Moran et al. 2013, Kerner et al. 2011, Jin et al. 2009, Murphy et al. 2008, Robinson et al. 2013, Robinson 2015, Fig. 1A).

Dicer is the widely conserved eukaryotic protein which cleaves dsRNA in the cytoplasm for the RNA interference pathway, also shows the C-terminal arrangement of 2 RNAselll and 1 dsRBD C-terminal domains strikingly similar to Drosha. The homology of these Drosha and Dicer C-terminal domains is widely accepted, and because of this similarity Drosha is taken to be result of a Dicer duplication. I (and colleague Dr. Ben Busby) have also observed putative Drosha-like BLAST hits in deeply branching eukaryotic taxa (unpublished data) and even in prokaryotes, however we did not resolve whether these represent contamination, horizontal gene transfer, results of longbranch attraction.

Meier et al. (2016) identified miRNAs and an apparent Drosha homolog (RbdB) in Dictyostelium discoideum. miRNA biogenesis function was shown by disruption and rescue by ectopic RbB expression, was necessary for production of the identified miRNAs. Additionally, Kruse et al. (2016) also showed RbdB has a functional nuclear localization domain, and interestingly that Dictyostelium Dicer homolog (DrnB) interacts with RbdB in the nucleolus. This may be representative of a functional Microprocessor in Unikonts, it is even suggestive of potential shared mechanism with the plant miRNA biogenesis mechanism, which utilizes a nuclear-localized Dicer (DCL1) for miRNA processing (Rogers and Chen, 2013). Dictyostelium miRNA processing therefore may provide evidence of an evolutionary origin for miRNA biogenesis at the base of Eukaryotes, much deeper than is usually hypothesized, and calling into question a true convergent evolution of bona fide miRNA biogenesis.

Recently published work of Brate et al., 2018, showed through genome and transcriptome sequencing and analysis of 6 species from the holozoan group Ichthyosporea, that 5 out of 6 species had identifiable Drosha and Pasha homologs, although bona fide miRNA was identified in only 1 of the species. They also identified the amino acid "bump" structure to be present, which is a key feature seen in the structure of Human Drosha (Kwon et al. 2016).

In Grimson et al. 2008, microRNAs in the demosponge Amphimedon queenslandica were reported, with a subset of 8 miRNAs conserved in a diversity of additional demosponge taxa (Wheeler et al. 2009). Among these conserved microRNAs, no orthologs were found for the many conserved Eumetazoan microRNAs or other poriferan classes (the calcisponges and homoscleromoph sponges) (Robinson et al. 2013). Both Grimson et al. 2008, Robinson et al. 2013, and Robinson 2015 report alignments of Drosha and Pasha sequences from the respective taxa. Findings of microRNAs in additional demosponges Stylissa carteri and Xestospongia testudinaria likewise report orthologous sequences for Drosha and Pasha (Liew et al. 2016).

Cnidarian miRNAs and Drosha and Pasha orthologs were identified in sequence data for Nematostella (Putnam et al. 2007) and Hydra (Chapman et al. 2010). An analysis of evolution of miRNA biogenesis in Metazoa including cnidarian taxa can also be found in Moran et al. 2013. Of interest is that cnidarian orthologs show more overall sequence similarity with bilaterian Drosha and Pasha than with other basal metazoan phyla, particularly in conservation of $\mathrm{N}$-terminal functional domains (vs. C-terminal RNAselll and dsRBD structural domains; Fig. 1A). This indicates that functional roles for the $\mathrm{N}$-terminal domains is likely conserved within Eumetazoa, while in the basal Metazoa and in Icthyosporea, sequence similarity of such $\mathrm{N}$-terminal regions remains questionable (although Brate et al. 2018 do show presence of Pasha WW domains, and lengthy Drosha N-terminal sequence in 
some Icthyosporea species, but truncation in others, Brate et al. 2016). Truncations in reported non-Eumetazoa sequence may also be due to incomplete sequence representation.

microRNA candidates are absent from placozoan Trichoplax adhaerens (Hertel et al. 2009), which has a Drosha but no Pasha ortholog (Srivastava et al. 2008). microRNA candidates are not found in ctenophore Mnemiopsis leidyi, nor are Drosha or Pasha orthologs (Maxwell et al. 2012). These support various scenarios of acquisition and loss, or basal absence depending on relative phylogenetic position of Ctenophora and Placozoa (Schierwater et al. 2016), yet all are consistent with the assumption that Drosha and Pasha orthologs represent presence of a canonical metazoan biogenesis pathway.

Presence of Drosha and Pasha orthologs with putative microRNAs is therefore reasonably standard criteria when identifying microRNAs in basal taxa outside the range of well-studied models as 'canonical microRNA'. Despite this, some authors have maintained convergent evolution of microRNA in phyla with Drosha and Pasha orthologs. In Robinson et al. 2013, reporting miRNAs in calcisponge and homoscleromorph sponges (Porifera), microRNA is presented as having had multiple independent origins with respect to eumetazoan miRNAs. In our discussion, we argued that while individual microRNAs are not specifically homologous (because of sequence dissimilarity), conservation of microRNA biogenesis processes in general is unclear and should be contingent on the experimental validation of microprocessor function to produce the observed miRNAs.

\section{Drosha, Pasha, and the Microprocessor complex perform many functions other than miRNA biogenesis.}

Canonical miRNAs were first discovered in the late $20^{\text {th }} /$ early $21^{\text {st }}$ century (Fire et al., 1998, Lau et al., 2001, Lee and Ambros, 2001). miRNAs originate from endogenously transcribed hairpins to regulate gene expression posttranscriptionally by targeting of partially complementary sequences. Proteins Drosha (vertebrate RNASEN) and Pasha (vertebrate DGCR8) were 'necessary and sufficient' for the recognition and cleavage of precursor stemloops from primary transcripts (pri-miRNA) during microRNA biogenesis (Gregory et al., 2004, Denli et al., 2004, Han et al., 2004). Pasha biochemistry showed that two C-terminal double-stranded RNA binding domains (dsRBD) facilitated recognition and binding of primary-miRNA by Pasha, and that the N-terminal domain of Pasha contained canonical WW (Tryptophan-Tryptophan) sequences (Landthaler et al., 2004, Yeom et al., 2006).

Drosha and Pasha are both known to perform non-miRNA functions. For example, Drosha was originally known as a ribosome biogenesis factor. The Drosha RNAse III domains structure and mechanism of cleavage are historically well-studied (Court et al., 2013). In Eumetazoans, Drosha possesses an N-terminal proline-rich domain, an arginine-serine rich domain, and a central, conserved domain of unknown function (DUF) (Wu et al., 2000, Lee et al., 2003, Han et al., 2006). While functional roles of the Drosha N-terminal P-rich, SR-rich domains are not as well understood, proline-rich and arginine-serine rich domains function in protein-protein interaction and spliceosomal interactions, respectively, in other protein families (Shepard and Hertel, 2009, Long and Caceres, 2009, Kay et al., 2000).

Although the structure and function of the central Drosha DUF is unknown, the N-terminal RS and Proline rich domains of vertebrate Drosha are shown to associate with the promoter to regulate transcription independently of its miRNA cleavage function (Gromak et al., 2013). Furthermore, Drosha has miRNA independent functions in mRNA cleavage and rRNA processing (Johanson et al., 2013), and may have roles in regulation of splicing (Havens et al., 2014). Arginine-serine (RS) domains are found in a large class of spliceosomal regulators (Shepard and 
Hertel, 2009, Long and Caceres, 2009); therefore, this domain in Drosha may play a role in the spliceosome associated miRNA processing or even represent a miRNA independent spliceosomal role for the microprocessor (Agranat-Tamir et al., 2014, Kataoka et al., 2009). That the N-terminal portion of the DUF overlaps with part of the vertebrate RS-domain may show that highly conserved part of this domain has some function in these processes, for example, it is possible that the vertebrate-specific proline domain facilitates an interaction with promoter elements not present in invertebrates. Conserved central DUF in Drosha plays a role in miRNA or other regulatory functions must be determined experimentally, it does not appear to play a role in establishing conserved miRNA complements, as it is present in Sycon but not Leucosolenia, calcisponge species that have been shown to possess at least one conserved miRNA (Robinson, 2015). Functional transcriptomics studies have elucidated many novel interactions for Pasha not clearly related to its miRNA functions (Macias et al. 2012, Kadener et al. 2009)

Pasha contains a heme-binding domain, required for miRNA processing and recognition of pri-miRNAs. Its associated WW domain facilitates dimerization of Pasha in the binding of heme (Faller et al., 2007, Senturia et al., 2010, Faller et al., 2010, Weitz et al., 2010, Quick-Cleveland et al., 2014). This structure-function relationship was even show experimentally to be conserved in Deuterostome invertebrates (Senturia et al., 2012) (Fig. 1B).

\section{A strict structural definition for "bona fide microRNA" begs the question of a structure-function relationship.}

Uncertainty in parsing small ncRNA in disparate non-eumetazoan taxa have led research groups to propose and implement nomenclature schemes to describe and define miRNAs and the diversity of non-canonical small noncoding regulatory RNA found in organisms. Newer schemes have placed effort on providing a naturalistic framework reflective of underlying evolutionary processes than the original database MiRBase (Griffiths-Jones et al. 2006).

A rigorously exclusive definition for 'bona fide microRNA' has also been implemented (Fromm et al. 2015), which have reduced by over half the number of microRNAs described as such in miRBase. Criteria for defining a bona fide microRNA are provided and elaborated upon, these include origin from genomic loci forming specific transcribed hairpin structure, location of the mature miRNA sequence in a specific orientation on the hairpin, and evidence of a paired duplex representing the complementary 'mate' (the 'star' sequence ie. miR-X, miR-X*) of the functionally mature microRNA. Additional meta-criteria are formalized in Fromm et al. 2015, which place value on conservation of sequence parameters for individual microRNA genes between species.

A limitation for such definitions is that the structure-function relationship must be inferred, for example so that while the defined miRNA presumably represents a 'bona fide' end product of an evolutionarily conserved mechanism, the relationship between the structure and functional molecular mechanism producing it remains poorly understood in its context. Bona fide microRNAs have been shown in eumetazoans, plants, and brown algae, for example, however these are clearly paraphyletic and therefore do not represent a conserved processing mechanism despite their grouping as 'bona fide'. (Tarver et al. 2015)

Desvignes et al. 2015 present a broad nomenclature describing an inclusive diversity of small noncoding RNAs, such as IncRNAs, miRtrons, endogenous siRNAs. The authors include the spectrum of noncoding RNA species ('gene level'), but also provide a classification based on downstream processing of the transcribed RNA ('precursor level'). These provide a high-level hierarchy of molecular mechanism, a fair advancement when 
attempting to contextualize related, diverse ncRNA origins and processing pathways. An inconsistency introduced in this scheme however, is that all end-product small ncRNAs are described as 'miRNAs' regardless of the origin of the precursor duplex. The broad sweep of small ncRNAs would more appropriately as 'short noncoding regulatory RNAs, rather than microRNAs which are historically defined according to the criteria above. Budak et al. 2016 for example also describe the need for a nomenclature of post-transcriptional miRNA modifications and non-canonical miRNA species.

\section{The cytoplasmic effector functions of bona fide microRNAs require the conserved eukaryotic RNAi machinery of Dicer and Argonaut.}

Dicer and Argonaute proteins are the cytoplasmic effectors present and capable of facilitating RNAi from double stranded RNA sources, in almost every eukaryotic taxa, and appear to be an ancient mechanism against viral parasitism (Shabalina and Koonin, 2012).

microRNA was discovered in the model organisms, nematode worm C. elegans and green plant Arabidopsis. In elucidating the molecular mechanism responsible for miRNA biogenesis, it was determined that green plants (ie. Streptophyta) and Eumetazoa produced microRNAs via different molecular mechanisms. This is taken as indication that miRNA biogenesis in plants and animals are of independent evolutionary origin.

It is something of an oversimplification however: plant and animal microRNA pathways do share common components: both plant and animal microRNA require the conserved eukaryotic RNA interference (RNAi) machinery for their effector function. This is principally the Dicer protein, an RNase III enzyme performing cytosolic cleavage of dsRNA, and the Argonaute proteins, which provide the enzymatic functions of targeting and transcript regulation for the microRNA itself. Both are found so universally, that it is clearly molecular synapomorphy in the eukaryotes, while it is a Dicer duplication that facilitates nuclear pri- to pre- processing in the plant nucleus. Even many of those taxa which lack microRNA under the standard definition, are able to effect RNAi functionality through experimental introduction of dsRNA, due to Dicer and Ago.

In the context of the 'homology' and 'convergence' labels it is therefore incorrect to describe 'microRNA' (ie. the total manifestation of the microRNA biogenesis pathway) as a biological entity wholly separate from RNAi, even though conserved microRNA loci and individual protein members could be described as homologs and orthologs. More accurately, 'canonical eumetazoan microRNA' can be described as a derived and specialized subset of RNA interference; novel in terms of the specific origin of microRNA transcripts and Drosha/Pasha biogenesis machinery, yet also dependent upon and derived from pre-existing functional pathways.

\section{Conclusion}

My goal has been to highlight three philosophical issues on the topic of "evolution of microprocessor-associated miRNA biogenesis".

First is that, while Drosha and Pasha homologs are found in various taxa, they may not add up to 'canonical miRNA biogenesis' due to the fact that $\mathrm{N}$-terminal protein-protein interaction domains and other domains found in Eumetazoa may not be present in lower, non-Eumetazoan taxa. Functional interactions of the Drosha Nterminal protein-protein interaction domains may have gained, or lost sequence and function based on adaptive 
evolutionary pressures, as it appears to have done, for example, in Placozoa (Fig. 2). On the other hand, experimental validation of the miRNA biogenesis function of putative Drosha homolog RbdB and nuclearlocalized Dicer homolog DrnB in Dictyostelium (Meier et al. 2016, Kruse et al. 2016), provides a clear basis for an assertion that holozoan/metazoan microprocessors may be derivative of an ancestral eukaryotic Dicer-based nuclear miRNA-producing machinery.

Second is that, categorization of miRNAs based on structural components of the RNA itself may result in paraphyletic grouping, for example as in the case of plant, animal, and brown algal miRNAs as 'bona fide', nonetheless having different, or convergently evolved biogenesis resulting in structural similarities not representative of evolutionarily conserved mechanics.

Finally, that ancient conserved cytoplasmic RNAi machinery in the Eukaryotes, is still used, with modification, to effect gene regulation via the canonical eumetazoan miRNA pathway. Canonical eumetazoan miRNA therefore cannot be totally separated from the Dicer/Ago functionality. Nuclear processing of pri- to pre-miRNA in eumetazoans is portrayed as residing in the novelty of the 'microprocessor'. Yet, the 'microprocessor' entity is really more of an ad-hoc association of deeply conserved RNAselll and dsRBD domain proteins, with proteinprotein interaction network having evolved in a stepwise to gain multiple specific functions along the evolutionary trajectory in Eumetazoa. This rather than being representative of a single de novo origin of novel miRNA biogenesis due to duplication or appearance of a novel RNAselll representative (ie. Drosha).

In conclusion, continuation of such research provides important foundation for understanding the likelihood and frequency, and molecular trends involved in evolving a complex and sophisticated molecular pathway such as miRNA biogenesis.

\section{References}

Agranat-Tamir, L. et al. 2014. Interplay between pre-mRNA splicing and microRNA biogenesis within the suprasplicosome. Nucleic Acids Research. 42(7):4640-4651.

Brate, J. et al. 2018. Unicellular Origin of the Animal MicroRNA Machinery. Current Biology. 28(20):P3288-3295. Doi: https://doi.org/10.1016/j.cub.2018.08.018.

Budak, et al. 2016. MicroRNA nomenclature and the need for a revised naming prescription. Brief Funct Genomics. 15(1):65-71.

Chapman, JA. et al. 2010. The dynamic genome of Hydra. Nature. 464:592-596.

Court, DL. et al. 2013. RNase III: Genetics and Function; Structure and Mechanism. Annual Reviews Genetics. 47:405-431.

Denli, AM. et al. 2004. Processing of primary microRNAs by the Microprocessor complex. Nature. 432:231-235.

Desvignes, T. et al. 2015. miRNA Nomenclature: A View Incorporating Genetic Origins, Biosynthetic Pathways, and Sequence Variants. Trends Genet. 31(11):613-626.

Faller, M. et al. 2007. Heme is involved in microRNA processing. Nature Structural \& Molecular Biology. 14(1):2329. 
Faller, M. et al. 2010. DGCR8 recognizes primary transcripts of microRNAs through highly cooperative binding and formation of higher-order structures. RNA. 16:1570-1583.

Fire, A. et al. 1998. Potent and specific genetic interference by double-stranded RNA in Caenorhabditi elegans. Nature. 391:806-811.

Fromm B. et al. 2015. A Uniform System for the Annotation of Vertebrate microRNA Genes and the Evolution of the Human microRNAome. Annual Reviews of Genetics. 49:213-242.

Gregory, RL. et al. 2004. The Microprocessor complex mediates the genesis of microRNAs. Nature. 432:235-240. Grimson, A. et al. 2008. Early origins and evolution of microRNAs and Piwi-interacting RNAs in animals. Nature 455, 1193-1197.

Gromak, N. et al. 2013. Drosha Regulates Gene Expression Independently of RNA Cleavage Function. Cell Reports. 5:1499-1510.

Han, J. et al. 2004. The Drosha-DGCR8 complex in primary microRNA processing. Genes \& Development. 18:3016-3027.

Han, J. et al. 2006. Molecular Basis for the Recognition of Primary microRNAs by the Drosha-DGCR8 Complex. Cell. 125:887-901.

Havens, MA. et al. 2014. Drosha Promotes Splicing of a Pre-microRNA-like Alternative Exon. PLOS Genetics. 10(5):e1004312.

Hertel, J. et al. 2009. Non-coding RNA annotation of the genome of Trichoplax adhaerens. Nucleic Acids Research. 37(5):1602-1615.

Jin, L. et al. 2009. The evolutionary study of small RNA-directed gene silencing pathways by investigatin RNase III enzymes. Gene. 435:1-8.

Johanson, TM. et al. 2013. MicroRNA-independent roles of the RNase III enzymes Drosha and Dicer. Open Biology. 3:130144.

Kadener, S. et al. 2009. Genome-wide identification of targets of the drosha-pasha/DGCR8 complex. RNA. 15:537-545.

Kataoka, N. et al. 2009. Functional Association of the Microprocessor complex with the Spliceosome. Molecular and Cellular Biology. 29(12):3243-3254.

Kay, BK. et al. 2000. The importance of being proline: the interaction of proline-rich motifs in signalling proteins with their cognate domains. The FASEB Journal. 14:231.

Kerner, P. et al. 2011. Evolution of RNA-Binding Proteins in Animals: Insights from Genome-Wide Analysis in the Sponge Amphimedon queenslandica. Molecular Biology and Evolution. 28(8):2289-2303.

Kruse, J. et al. 2016. The protein domains of the Dictyostelium microprocessor that are required for correct subcellular localization and for microRNA maturation. RNA Biol. 13(10):1000-1010. 
Kwon et al. 2016. Structure of Human DROSHA. Cell. 164(1-2):81-90.

Landthaler, M. et al. 2004. The Human DiGeorge Syndrome Critical Region Gene 8 and Its D. Melanogaster Homolog Are Required for miRNA Biogenesis. Current Biology. 14:2162-2167.

Lau, NC. et al. 2001. An Abundant Class of Tiny RNAs with Probably Regulatory Roles in Caenorhabditis elegans. Science. 294:858-861.

Lee, RC., Ambros, V. 2001. An Extensive Class of Small RNAs in Caenorhabditis elegans. Science. 294:862-863.

Lee, Y. et al. 2003. The nuclear RNase III Drosha initiates microRNA processing. Nature. 425:415-419.

Liew, YJ. et al. 2016. miRNA Repertoires of Demosponges Stylissa carteri and Xestospongia testudinaria. PLoS One. 11(2):e0149080.

Long, JC., Caceres, JF. 2009. The SR protein family of splicing factors: master regulators of gene expression. Biochemical Journal. 417:15-27.

Macias, S. et al. 2012. DGCR8 HITS-CLIP reveals novel functions for the Microprocessor. Nature Structural Molecular Biology. 19(8):760-766.

Maxwell, et al. 2012. MicroRNAs and essential components of the microRNA processing machinery are not encoded in the genome of the ctenophore Mnemiopsis leidyi. BMC Genomics. 13:714.

Meier, D. et al. 2016. Analysis of the Microprocessor in Dictyostelium: The Role of RbdB, a dsRNA Binding Protein. PLoS Genetics. 12(6):e1006057.

Moran, Y. et al. 2013. The Evolution of MicroRNA Pathway Protein Components in Cnidaria. Molecular Biology and Evolution. 30(12):2541-2552.

Murphy, D. et al. 2008. The evolution of core proteins involved in microRNA biogenesis. BMC Evolutionary Biology. 8:92.

Putnam, NH. et al. 2007. Sea Anemone Genome Reveals Ancestral Eumetazoan Gene Repertoire and Genomic Organization. Science. 317(5834):86-94.

Quick-Cleveland, J. et al. 2014. The DGCR8 RNA-Binding Heme Domain Recognizes Primary MicroRNAs by Clamping the Hairpin. Cell Reports. 7:1994-2005.

Robinson, JM, et al. 2013. The identification of microRNAs in calcisponges: independent evolution of microRNAs in basal metazoans. Journal of Experimental Zoology B: Molecular Development and Evolution. 320(2):84-93.

Robinson, JM. 2015. microRNA in Porifera: Evolution of a Molecular Mechanism in Sponges. PhD dissertation. Dartmouth College. Ann Arbor: ProQuest/UMI, 2015. (Publication No. 3685076).

Rogers, K., Chen, X. 2013. Biogenesis, turnover, and mode of action of plant microRNAs. Plant Cell. 25(7):238399.

Schierwater, B. et al. 2016. Never Ending Analysis of a Century Old Evolutionary Debate: "Unringing" the Urmetazon Bell. Frontiers in Ecology and Evolution. 4(5): 
Senturia, R., Faller, M., Yin, S., Loo, JA., Cascio, D., Sawaya, MR., Hwang, D., Clubb, RT., Guo, F. 2010. Structure of the dimerization domain of DiGeorge Critical Region 8. Protein Science. 19:1354-1365.

Senturia R, et al. 2012. Dimerization and Heme binding Are Conserved in Amphibian and Starfish Homologues of the microRNA Processing Protein DGCR8. 7(7):e39688.

Shabalina, SA, Koonin, EV. 2012. Origins and evolution of eukaryotic RNA interference. Trends in Ecology and Evolution. 23(10):578-587.

Shepard, PJ., Hertel, KJ. 2009. The SR protein family. Genome Biology. 10:242.

Srivastava, et al. 2008. The Trichoplax genome and the nature of placozoans. Nature. 454(7207):955-60.

Tarver, JE. et al. 2012. Do miRNAs have a deep evolutionary history? Bioessays. 34(10):857-866.

Tarver, JE. et al. 2015. microRNAs and the evolution of complex multicellularity: identification of a large, diverse complement of microRNAs in the brown alga Ectocarpus. Nucleic Acids Research. 43(13):6384-6398.

Weitz, SH. et al 2014. Processing of microRNA primary transcripts requires heme in mammalian cells. PNAS. 111(5):1861-1866.

Wheeler, BM. et al. 2009. The deep evolution of metazoan microRNAs. Evolution \& Development. 11(1)50-68.

Wu, H. et al. 2000. Human RNase III Is a 160-kDa Protein Involved in Preribosomal RNA Processing. The Journal of Biological Chemistry. 275(47):36957-36965.

Yeom, KH. et al. 2006. Characterization of DGCR8/Pasha, the essential cofactor for Drosha in primary miRNA processing. Nucleic Acids Research. 34(16):4622-4629.

\section{Figures}

Figure 1. A. Domain architecture comparison of metazoan Drosha orthologs. Note that the RNAselll and dsRBD domains are highly conserved, while $\mathrm{N}$-terminal regions of PfamB motifs were not identified, or highly modified, in sponges. (Human RNAselll image adapted from Wu et al. 2000). B. Domain architecture comparison of metazoan Pasha orthologs. Note that calcisponges Leucosolenia and Sycon do possess the canonical Pasha heme sequence, which is missing and therefore inferred lost in demosponge Amphimedon. (top alignment performed with JalView using sequences accessions in supplementary section below; middle alignment and bottom diagram adapted from Senturia et al. 2012). 
A.

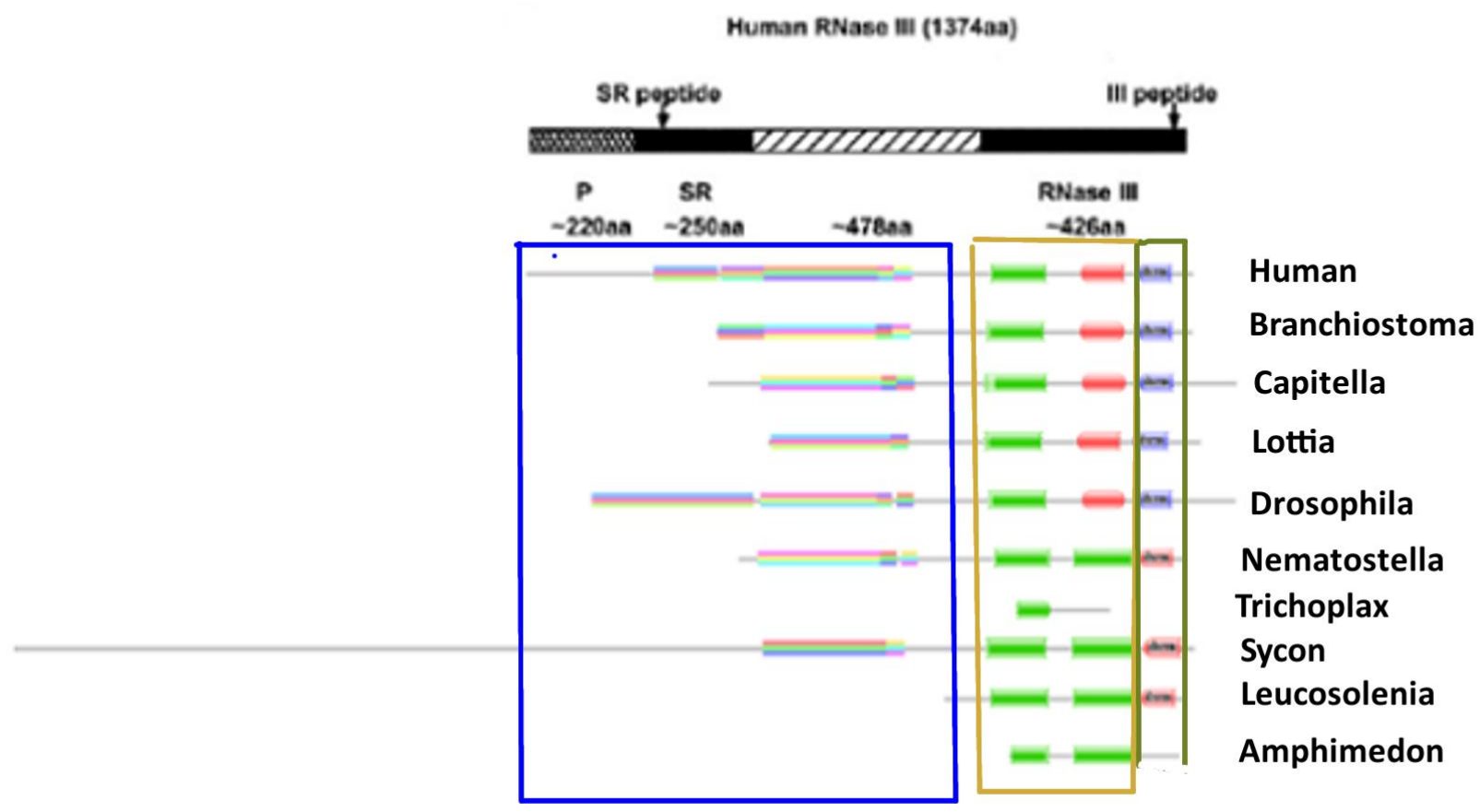


B.

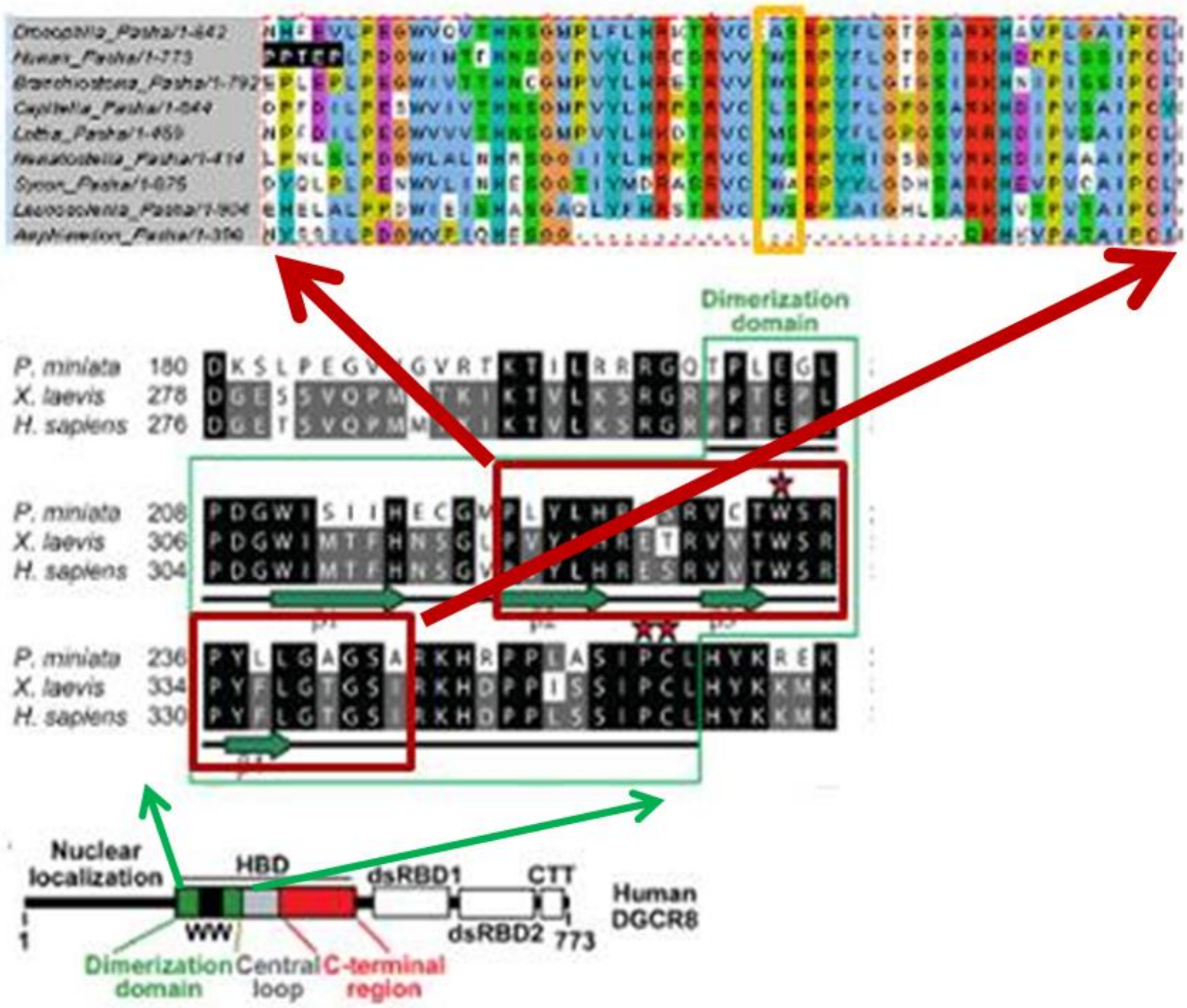


Figure 2. Cladogram describing current knowledge of microprocessor evolution within Holozoa.

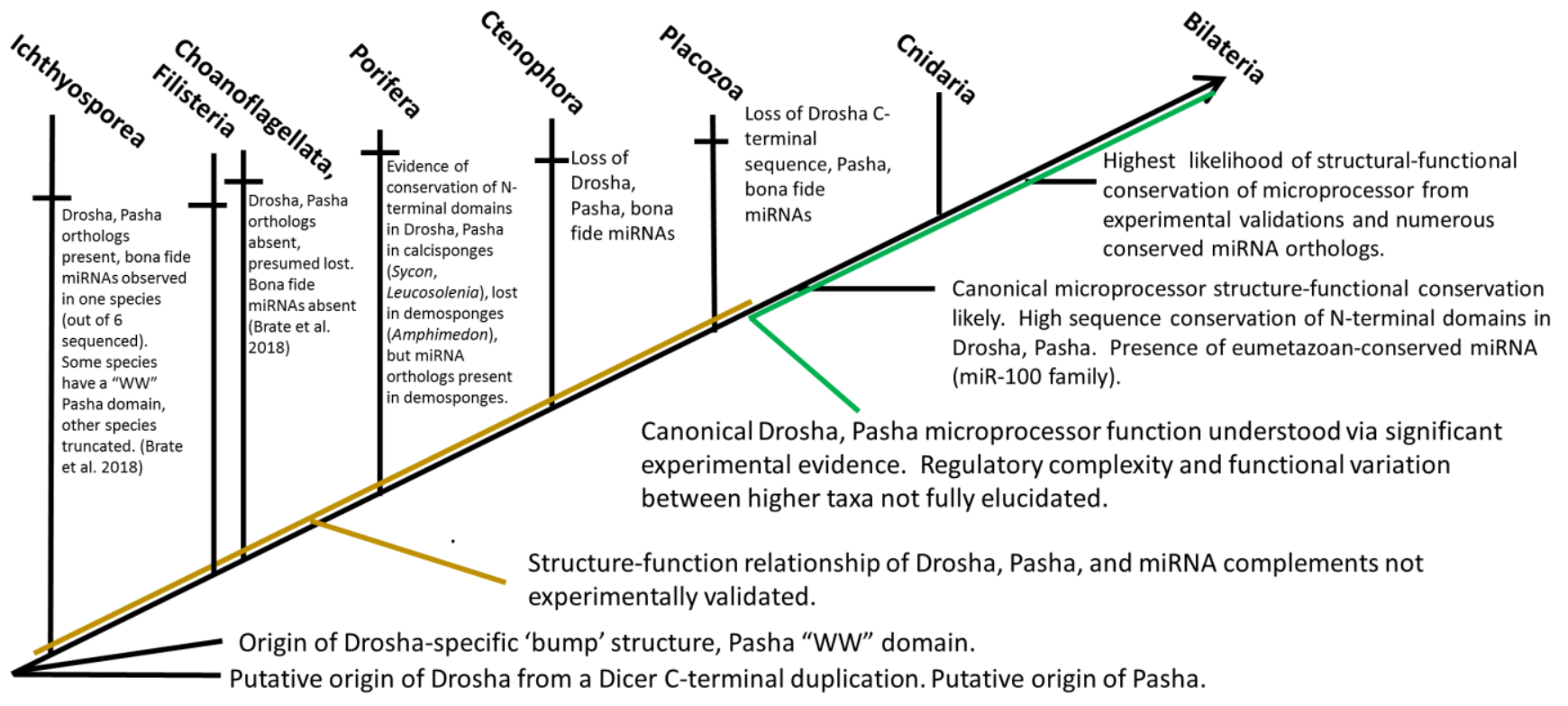

Scenario 1: 'bona fide' miRNAs are produced by a Drosha-Pasha microprocessor. Presence of Drosha in the holozoan LCA represents an ancestral holozoan microprocessor. $\mathrm{N}$-terminal functional sequence evolves subsequently, providing novel regulatory interaction and context specificity for miRNA biogenesis in Eumetazoa.

Scenario 2: Drosha which performs generalized RNAselll function, and is not necessarily complexed with Pasha as the functional "microprocessor" of canonical eumetazoan miRNAs, although Drosha may still produce bona fide miRNAs in 'lower' holozoans. 


\section{Supplementary Data. Sequence accession data (2015).}

\section{NCBI Protein database (http://www.ncbi.nlm.nih.gov/protein)}

Drosophila (Arthropoda):

>gi|17137682 |ref|NP_477436.1| drosha [Drosophila melanogaster]

Human (Vertebrata):

>gi|20139357|sp|Q9NRR4.2|RNC_HUMAN RecName: Full=Ribonuclease 3; AltName: Full=Protein Drosha;

AltName: Full=Ribonuclease III; Short=RNase III; AltName: Full=p241

Amphimedon (Demospongia):

>gi|340378479|ref|XP_003387755.1| PREDICTED: ribonuclease 3-like [Amphimedon queenslandica]

\section{JGI (http://genome.jgi.doe.gov/)}

Branchiostoma floridae (Cephalochordata), v1.0 Filtered Gene Models (proteins)

>jgi|Brafl1|133907|gw.567.1.1

Capitella capitata (Annelida), v1.0 Filtered Gene Models

>jgi|Capca1|165112|estExt_Genewise1.C_70199

Lottia gigantea (Mollusca), v1.0 filtered model proteins (Lotgi1_GeneModels_FilteredModels1_aa)

>jgi|Lotgi1|230088|estExt_fgenesh2_pg.C_sca_100341

Trichoplax adhaerens (Placozoa), v1.0 best proteins

>jgi|Triad1|20536|e_gw1.2.1514.1

Nematostella vectensis (Cnidaria), v1.0 models (proteins)

>jgi|Nemve1|10066| gw.302.2.1

\section{SARS Sycon/Leucosolenia protein predictions (http://sycon.sars.uib.no/sycon):}

Sycon ciliatum (Calcarea), proteins database

>13204 scpid5641| scgid13951| Ribonuclease 3; Protein Drosha; Ribonuclease III

Leucosolenia (Calcarea), proteins likely including contaminants database

>31099 Icpid74715| Icgid31389| Ribonuclease 3; Protein Drosha; Ribonuclease III; p241

\section{Pasha sequences}

NCBI:

Drosophila (Arthropoda) 
>gi|7302074|gb|AAF57175.1| partner of drosha, isoform A [Drosophila melanogaster]

Human (Vertebrata)

>gi|38488720|ref|NP_073557.3| microprocessor complex subunit DGCR8 isoform 1 [Homo sapiens]

JGI:

Branchiostoma floridae (Cephalochordata), v1.0 Filtered Gene Models (proteins)

>jgi|Brafl1|97363|fgenesh2_pg.scaffold_302000018

Capitella capitata (Annelida), v1.0 Filtered Gene Models

>jgi|Capca1|219105 | estExt_fgenesh1_pg.C_5460013

Lottia gigantea (Mollusca), v1.0 filtered model proteins (Lotgi1_GeneModels_FilteredModels1_aa)

>jgi|Lotgi1|141857|e_gw1.169.2.1

Ensembl Metazoa predicted protein:

Amphimedon queenslandica (Demospongia)

$>$ Aqu1.229085

https://metazoa.ensembl.org/Amphimedon queenslandica/Transcript/Sequence Protein?db=otherfeatures;g=A

qu1.229085; r=Contig13518:363522-367026;t=PAC:15727613

SARS Sycon/Leucosolenia protein predictions:

Sycon ciliatum (Calcarea), proteins database

>40029 scpid40203 | scgid4742| Microprocessor complex subunit DGCR8; DiGeorge syndrome critical region 8 homolog

Leucosolenia (Calcarea), proteins likely including contaminants database

>85279 Icpid31223| Icgid2003| Microprocessor complex subunit DGCR8; DiGeorge syndrome critical region 8 homolog; Gy1 